\title{
Entre el desarraigo y la querencia. Jóvenes rurales y TIC en Uruguay. Una aproximación cualitativa.
}

\author{
Rosario Sánchez Vilela \\ Universidad Católica del Uruguay - Montevideo - Uruguay \\ ORCID: https://orcid.org/0000-0003-2995-0520 \\ Celeste Borjas \\ Universidad Católica del Uruguay - Montevideo - Uruguay \\ ORCID: https://orcid.org/0000-0002-2438-7057
}

\begin{abstract}
Resumen
Los estudios sobre acceso y uso de TIC se han concentrado fundamentalmente en el país urbano mientras que los jóvenes rurales han sido escasamente abordados en su especificidad. Esta investigación se propuso una aproximación a la comprensión de este sector poblacional en Uruguay con el fin de contribuir a su visibilidad en el espacio público. Se exponen aquí los resultados del estudio cualitativo de un grupo de jóvenes rurales uruguayos, compuesto por estudiantes de bachillerato agrario de la Escuela Agraria de Montes. El recorrido que proponemos parte de un panorama general sobre el lugar de los jóvenes en la población uruguaya y el acceso a las tecnologías de la comunicación, así como la problematización de las categorías de juventud y ruralidad. El diseño metodológico adopta un enfoque socioantropológico en busca de significados más que de impactos, utilizando la entrevista cualitativa. En el análisis se exploran dimensiones espaciales y temporales de la cotidianidad -los tiempos de ocio, estudio y trabajo-, la sociabilidad, las representaciones e imaginarios sobre estas tecnologías y las proyecciones sobre el futuro. Los resultados de la investigación permiten identificar algunas claves para comprender las necesidades de este grupo poblacional. Las relaciones que se establecen en torno a las TIC, se presentan de forma ambigua y conflictiva. Su clave interpretativa radica en una situación vital atravesada por la tensión entre la expectativa inminente de migración a la ciudad y el deseo del retorno al ámbito rural y familiar.
\end{abstract}

Palabras clave: Jóvenes. Ruralidad. TIC.

\section{Between rootlessness and attachment. Rural youth and ICTs in Uruguay. A qualitative approximation.}

\section{Abstract}

Studies on access and use of ICTs have been mainly focused on the urban country. On the contrary, rural youth have been poorly considered in their specificity. The objective of this research was to present an approximation to the understanding of rural youth in Uruguay in order to contribute to their visibility on the public space. In this article, the results of a 
qualitative study of a group of rural Uruguayan youth, made up of ten high school students of Montes Agrarian School, are presented. The route that we propose begins with the presentation of a general panorama on the place of young people in the Uruguayan population and the access to communication technologies as well as the discussion of the categories of youth and rurality. The methodological design adopts a socio-anthropological approach in search of meanings rather than impacts, through the implementation of the qualitative interview. In the analysis, special and temporal dimensions of everyday life are explored-; leisure, study and work times-; sociability and representations and imaginaries about these technologies and projections about the future. The results of the research allow us to identify some keys to understand the needs of this population group. The relationships that are established around ICTs are presented in an ambiguous and conflictive way. The key to interpret them lies in a vital situation crossed by the tension between the imminent expectation of migration to the city and personal growth and the desire to return to the rural and family environment.

Keywords: Youth. Rurality. ICTs.

\section{Entre o desenraizamento e a querência. Juventude rural e TIC no Uruguai. Uma abordagem qualitativa.}

\section{Resumo}

Os estudos sobre o acesso e o uso das TICs concentraram-se principalmente no campo urbano, enquanto a juventude rural quase não foi abordada em sua especificidade. Esta pesquisa propôs uma abordagem para a compreensão desse setor populacional no Uruguai a fim de contribuir para sua visibilidade no espaço público. Apresentam-se aqui os resultados do estudo qualitativo de um grupo de jovens rurais uruguaios, composto por alunos do ensino médio agrário da Escola Agrária de Montes. O percurso que propomos parte de um panorama geral sobre a inserção dos jovens na população uruguaia e o acesso às tecnologias de comunicação, bem como a problematização das categorias juventude e ruralidade. $O$ desenho metodológico adota uma abordagem socioantropológica em busca de significados e não de impactos, por meio de entrevista qualitativa. A análise explora dimensões espaciais e temporais da vida cotidiana - tempos de lazer, estudo e trabalho -, sociabilidade, representações e imaginários sobre essas tecnologias e projeções sobre o futuro. Os resultados da pesquisa permitem identificar algumas chaves para compreender as necessidades deste grupo populacional. As relações que se estabelecem em torno das TIC são apresentadas de forma ambígua e conflituosa. Sua chave interpretativa reside em uma situação vital atravessada pela tensão entre a expectativa iminente de migração para a cidade e o desejo de retorno ao meio rural e familiar.

Palavras-chave: Juventude. Ruralidade. TIC.

\section{Introducción}

Esta comunicación se propone exponer los resultados de una investigación cuyo objetivo general fue estudiar la relación entre jóvenes, tecnología y ruralidad en los jóvenes rurales en el Uruguay, mediante el estudio de un caso acotado: un grupo de jóvenes que asisten a la Escuela Agraria de Montes ${ }^{1}$.

Según el Informe de la Unión Internacional de Telecomunicaciones (UIT, 2017), Uruguay es el país latinoamericano mejor posicionado en el Índice de

\footnotetext{
${ }^{1}$ Pequeña población de 1760 habitantes al noreste del Departamento de Canelones, Uruguay. Si bien la Escuela Agraria está asentada allí, ninguno de sus habitantes forma parte del alumnado de la Escuela Agraria.
} 
Desarrollo de las Tecnologías de la Información y la Comunicación. ${ }^{2}$ Sin embargo, esta posición favorable del país a nivel regional, en cuanto a la introducción y aprovechamiento de las nuevas tecnologías para el desarrollo, no exime de la necesidad de implementar políticas de inclusión digital que, más allá del acceso, promuevan el empleo de las nuevas tecnologías para el desarrollo de capacidades en comunidades en situación de desventaja social (CASAMAYOU, 2012, 2016).

El incremento de equipos y conectividad extendida, además de la implementación del plan $\mathrm{Ceibal}^{3}$, ha tenido como resultado la disminución de la brecha de acceso digital. No obstante, los procesos de apropiación de las TIC se asocian no solo al acceso, sino que conciernen a la capacidad del usuario de otorgarles un significado relevante en su vida cotidiana, que las vincule directamente con sus necesidades, motivaciones e intereses más cercanos (WINOCUR, SÁNCHEZ VILELA, 2016). Si bien los jóvenes son el sector de la población que más utiliza las TIC, su acceso y uso no son necesariamente uniformes, sino que pueden tener distintos significados y experimentar diversos procesos de apropiación. El conocimiento de estas diferencias, resulta esencial para la definición de políticas públicas tendientes a promover la inclusión y el desarrollo.

Los estudios sobre acceso y uso de TIC se ha concentrado fundamentalmente en el país urbano y los jóvenes rurales han sido una población escasamente abordada en su especificidad. Nuestro trabajo tiene el propósito de lograr una aproximación a su comprensión y contribuir a su visibilidad en el espacio público.

El recorrido que proponemos parte de un panorama general sobre el lugar de los jóvenes en la población uruguaya y el contexto de acceso a las tecnologías de la comunicación, para exponer después la perspectiva teórico-metodológica. Esa perspectiva supone la problematización de las categorías de juventud y ruralidad y el diseño metodológico adopta un enfoque socioantropológico en busca de significados más que de impactos. Por último, se exponen algunos de los resultados de la investigación en los que se pueden identificar algunas claves para comprender las necesidades de este grupo poblacional.

\section{Algunos datos sobre los jóvenes en Uruguay y el acceso a las tecnologías de la comunicación.}

Uruguay tiene una población altamente envejecida, que no llega a los tres millones y medio de habitantes. De acuerdo al último censo, de 2011, la población

\footnotetext{
${ }^{2}$ El Measuring the information Society report del 2017 ubica a Uruguay en el primer lugar en América Latina, delante de Argentina y Chile.

${ }^{3}$ El Plan Ceibal ("Conectividad Educativa de Informática Básica para el Aprendizaje en Línea" es un proyecto socioeducativo de Uruguay) fue creado por decreto del 18 de abril de 2007 "con el fin de realizar estudios, evaluaciones y acciones, necesarios para proporcionar un computador portátil a cada niño en edad escolar y a cada maestro de la escuela pública, así como también capacitar a los docentes en el uso de dicha herramienta, y promover la elaboración de propuestas educativas acordes con las mismas".' Presidencia de la República. Decreto 144/007. Disponible en: http://web.archive.org/web/20140630034353/http://archivo.presidencia.gub.uy/_Web/decretos/2007/ 04/EC579_18\%2004\%202007_00001.PDF. Consultado el 30 noviembre de 2015.
} 
joven (entre 14 y 29 años) llega a 777.104 personas, lo que representa el $23 \%$ de la población total. En ese segmento poblacional el 27,5\% tiene de 14 a 17 años; el 29,1\% tiene de 18 a 24 años y el $43,4 \%$ de 25 a 29 años y en proporciones muy similares de hombres y mujeres, 51,1\% y 49,9\% respectivamente (CALVO, 2014, p.10). Dentro de Uruguay, las regiones con mayor porcentaje de jóvenes son, en orden descendente, los departamentos de: Montevideo, Canelones, Maldonado, Salto y Paysandú (p.15). Del total de jóvenes en Uruguay un 5,1\% se considera como población dispersa, un $5,5 \%$ se encuentra en localidades de menos de 2000 habitantes y un $4,7 \%$ en localidades entre 2000 y 5000 habitantes. Al contemplar esa misma población vinculada particularmente al tema de educación, el porcentaje de jóvenes de poblaciones menores a 2000 habitantes, que declara estar estudiando, es considerablemente inferior al de las poblaciones de más de 5.000 personas. Esta diferencia se hace más notoria al considerar la franja etaria que se extiende entre los jóvenes de 20 a 29 años.

En cuanto al acceso a las tecnologías de la comunicación e internet en los jóvenes es necesario considerarla en el panorama general de expansión digital en el país. El conjunto de su población tiene un alto acceso a internet y las tecnologías de la comunicación asociadas. Según datos de la EUTIC ${ }^{4}$ para 2019 la cantidad de hogares con conexión a internet llegó a $88 \%$ y con banda ancha fija a $71 \%$. Además, la cantidad de usuarios de internet según este trabajo asciende a $87 \%$ y afirma que 9 de cada 10 uruguayos usan internet diariamente. El dispositivo preferido para la conexión es el celular: el $90 \%$ de quienes se conectan diariamente a internet lo hacen por ese medio (EUTIC, 2019). La brecha digital en su dimensión socioeconómica y generacional es reducida y continúa descendiendo. Según la misma encuesta, la brecha de acceso a internet entre los hogares de quintiles 1 y 5 se redujo a la mitad entre 2010 a 2019: la brecha entre los hogares con acceso a internet por quintil de ingreso era del 66\% en 2010 y fue de $29 \%$ en 2019. No obstante, como señala la encuesta WIP (DODEL, AGUIRRE, 2018) :"continúa existiendo una brecha de acceso importante en términos de nivel educativo y socioeconómico, donde los hogares de niveles altos duplican en acceso a los de más bajos" (p.12).

Internet y la radio son los medios más relevantes para los uruguayos en cuanto al entretenimiento y el tiempo libre. En los jóvenes entre los 18 y 29 años, el $42 \%$ usa la radio para su tiempo libre y el $59 \%$ internet. Además, el uso de internet en los menores de 30 años es casi universal en tanto alcanza el $98 \%{ }^{5}$ (DODEL, AGUIRRE, 2018, p.10-12).

Es necesario señalar que los datos de que se dispone a través de estos estudios y también de las encuestas oficiales ${ }^{6}$ muestran un panorama del país

\footnotetext{
${ }^{4}$ La Encuesta de Usos de Tecnologías de la Información y la Comunicación (EUTIC) es realizada por la Agencia de Gobierno Electrónico (Agesic) y el Instituto Nacional de Estadística (INE). Se realiza a partir de entrevistas personales con un integrante del hogar, población mayor de 14 años residente en Montevideo y el interior del país; muestra efectiva: 2341 hogares; período de relevamiento: setiembre y octubre de 2019.

${ }^{5}$ La misma encuesta revela que el porcentaje las personas de 60 años y más es de $56 \%$

${ }^{6}$ Nos referimos tanto a las encuestas el Ine referidas a toda la población (EUTIC 2013) como a aquellas centradas en los jóvenes (ENAJ 2013). Informe "Tercera Encuesta Nacional de Adolescencia y Juventud” -ENAJ 2013-. INJU-MIDES-MTSS_INE- ISEF_INM. Marzo de 2015. Disponible en:
} 
urbano, tomando en cuenta poblaciones de más de 5000 habitantes, por lo que las desigualdad de acceso y de calidad del acceso con respecto al contexto rural no ha sido medida con precisión.

Estas ausencias son parte de un fenómeno más general, como señaló oportunamente SUNKEL (2015):

Si bien el módulo de cultura encargado por la OEI ha permitido analizar los actuales consumos culturales juveniles, es importante resaltar la precariedad de las estadísticas sobre cultura. Debido a que en esta base de datos no existen series de tiempo, no es posible realizar análisis de tendencias ni desagregaciones por algunas variables sociodemográficas que permitan distinguir entre adolescentes y jóvenes, o entre consumos en el ámbito urbano y rural (p. 173).

La escasa producción, tanto en datos cuantitativos como en cualitativos, respecto de los usos de las TIC de este sector de la población habilita a cuestionarse, entonces, sobre el modo en que se vinculan con estas tecnologías en sus contextos específicos e indagar en las oportunidades y obstáculos que se presentan en la vida de estos jóvenes, para descifrar los significados que les atribuyen. El estudio de caso que aquí se presenta, intentó una aproximación a la experiencia de esta población en su relacionamiento con las TIC, desde un contexto sociocultural y material específico.

\section{Perspectiva teórico-metodológica.}

La categoría juventud ha sido largamente discutida en distintos campos disciplinares. Así, en el ámbito de los estudios culturales británicos se ha analizado críticamente su instalación como metáfora del cambio social y como categoría teórica que uniformiza y diluye las diferencias, particularmente la pertinencia de la diferencia de clase social (HALL y JEFFERSON, 1975-1993). En una dirección similar, se han desarrollado en los últimos años enfoques que intentan comprender la pluralidad de situaciones y que subrayan los diversos modos de ser joven (GARCÍA CANCLINI, 2012; REGUILLO, 2010).

Por otra parte, los estudios sobre juventud históricamente han estado permeados por la mirada del mundo adulto: es el adulto quien interpreta, decodifica y finalmente caracteriza al universo de los jóvenes, el cual es representado esencialmente como el futuro de la sociedad. Se desatiende consecuentemente, asuntos que dan cuenta del presente de los jóvenes y sus complejidades (FEIXA, C, OLIART, P (coords.); (2016.p.20). Este enfoque provoca una concepción e implementación de políticas sociales alejadas de las verdaderas necesidades e inquietudes de la población en cuestión, realidad especialmente apreciable al considerar a la población rural. No obstante, se vislumbra un sostenido intento de abandonar el enfoque adultocéntrico de la cultura juvenil y favorecer una perspectiva intergeneracional donde:

http://www.ine.gub.uy/documents/10181/66919/tercer-informe-encuesta-nacional-de-juventud2013.pdf/b34boab4-9409-4557-a62c-1f313e6420eb. Consultado 10 de abril 2016. 
(...) las reglas del juego son el resultado de la interacción entre generaciones-y culturocéntrica -la cultura es el área principal en la que se redefine el ámbito público y, por lo tanto, es el espacio para el surgimiento de nuevas identidades política (FEIXA, C, OLIART, P (coords.); (2016.p. 22).

La juventud no puede considerarse un grupo homogéneo en tanto abarca las múltiples experiencias que se viven en distintos entornos socioculturales. En esa dirección REGUILLO (2010) subraya que:

(...) existen dos juventudes: una mayoritaria, precarizada desconectada, no solo de la sociedad de la información, sino desconectada o desafiliada de las instituciones (educación, salud, trabajo, seguridad) sobreviviendo apenas con los mínimos y otra minoritaria conectada y en condiciones de elegir" (p. 432).

También Rüdiger (2016) recoge la perspectiva que atiende a la diversidad inherente a la realidad social:

No es posible definir a los jóvenes como un grupo homogéneo, sino como grupos juveniles que constituyen un conjunto heterogéneo, jóvenes con oportunidades, dificultades, facilidades y poder en las sociedades. En este sentido la juventud es una construcción social, es la producción de una determinada sociedad que se origina de varias formas, producción en la que se conjugan, entre otros factores, estereotipos, momentos históricos, múltiples referencias, además de diferentes y diversificadas situaciones de clase, género, etnia, grupo, etc (p.42).

En el tema que nos ocupa en este artículo se perfila, además, una distinción central: entre jóvenes urbanos y jóvenes rurales. Se tiende a pensar en los jóvenes asociados a los espacios urbanos, mientras que los jóvenes vinculados a ámbitos rurales no suelen ser objeto de estudio específico y consecuentemente se produce una cierta invisibilidad de este grupo social. Esta mirada estuvo alimentada a su vez, por una imagen del joven como desinteresado respecto al medio rural, lo que ha contribuido a cierta "invisibilidad de la categoría como formadora de identidades sociales y, por lo tanto, de demandas sociales "(CASTRO, et al, 2009, p.23). Es recién a partir del 2000 que surge la noción del joven rural como nuevo actor político (SERRANO, 2017, p.55; CASTRO et al 2009). La ruralidad ha estado impregnada, entonces, por procesos de invisibilización ${ }^{7}$, que se materializan históricamente, tanto a nivel regional como local:

(...) esta invisibilidad tiene como epicentro teórico un sesgo urbanizante en todo estudio acerca de lo rural, en la medida en que lo concibe como una instancia arcaica a ser superada a través del desarrollo modernizante; de ahí que no revista interés propio para ser pensado como objeto específico. Esto, sumado a una consideración de la juventud eminentemente urbana, da por resultado un escaso desarrollo de la juventud rural como tema autónomo de debate, discusión y políticas específicas, en particular si se compara con su homólogo urbano (GONZÁLEZ CANGAS, 2003).

El sesgo urbanizante supuso considerar lo rural como un mero constructo opuesto a la modernización industrial, propia de la ciudad. De esta manera, se minimizó el carácter complejo y heterogéneo inherente a las juventudes rurales, al mismo tiempo que emerge también una percepción de los jóvenes rurales como

\footnotetext{
${ }^{7}$ Así se manifiesta, por ejemplo, en el informe del año 2005, Estado del arte de las investigaciones sobre juventud rural en América Latina, en el que Gabriel Kessler señala un vacío teórico respecto a la categoría "juventud rural". Esta ausencia refiere no solo al nivel académico, sino de desarrollo de políticas públicas hacia el sector (DURSTON, 1997).
} 
futuros campesinos, continuadores de determinados estilos vida, a menudo asociados a lo estático, atrasado, anclados en la tradición y alejados de posibilidades reales de desarrollo y progreso (DURSTON,1996,1997; GONZÁLEZ CANGAS, 2003).

No obstante, al mismo tiempo que se constatan estas percepciones se ha producido el surgimiento de nuevas ruralidades (ESCOSTEGUY, 2019, p. 32-52), a partir de la expansión de la tecnología y la transformación de estilos de vida en los que se producen algunos sincretismos de lo rural y lo urbano. Para SERRANO (2017)

\begin{abstract}
(...) no se puede decir simplemente que hay una nueva ruralidad o nuevas ruralidades, sino múltiples ruralidades, donde representaciones modernas y representaciones contemporáneas conviven y se transforman constantemente, mostrando que las representaciones sobre la ruralidad son polisémicas y transitorias, por combinar representaciones modernas y contemporáneas, muchas veces en un mismo sujeto (p.206).
\end{abstract}

La identificación de ese carácter polisémico y transitorio al que refiere la autora, es el mismo que nos motiva a indagar sobre la realidad de los jóvenes rurales en el Uruguay, en el afán de ofrecer respuestas sobre los distintos modos de vivir la juventud y su relación con las tecnologías, que estos actores despliegan.

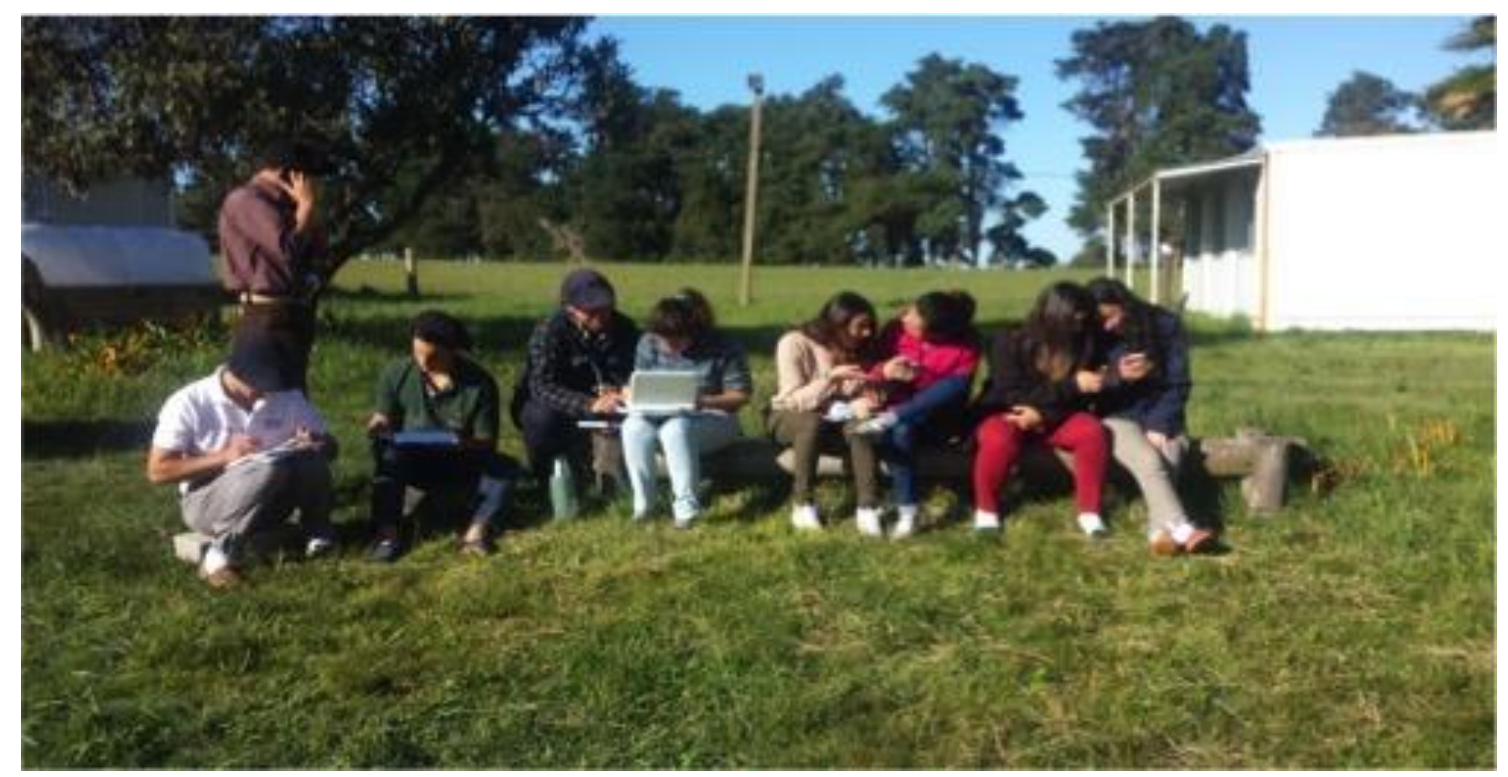

Escuela Agraria de Montes. Fuente: Celeste Borjas.

\title{
Diseño metodológico
}

La idea de fragmentación y diversidad de las identidades juveniles conduce a la constatación de que su comprensión requiere un enfoque múltiple (FEIXA, OLIART, 2016). El abordaje que aquí proponemos se inscribe en la línea de los estudios de las audiencias en sus contextos de recepción, en el entendido de que en los consumos y usos los jóvenes van constituyendo sus identidades y sus formas de sociabilidad (MORDUCHOWICZ, 2008 y 2012). En estas prácticas se expresan las nuevas sensibilidades, las tensiones y negociaciones de sentido, se definen los estilos de vida como clave de diferenciación y pertenencia.

Nos hemos propuesto un estudio orientado a comprender el consumo de las 
tecnologías de la comunicación como un proceso estrechamente vinculado a la producción de sentido inserto en la vida diaria de los sujetos, en una situación específica y no necesariamente generalizable.

Esta comunicación se concentra en exponer y analizar los resultados del estudio de un grupo de jóvenes rurales uruguayos compuesto por diez estudiantes de bachillerato agrario de la Escuela Agraria de Montes $^{8}$, seis mujeres y cuatro varones entre los 15 y 18 años, que viven en familias cuya subsistencia depende de las tareas rurales.

El diseño metodológico adoptó una perspectiva socio-antropológica para recuperar la experiencia de los sujetos y el modo cómo dan sentido práctico y simbólico en sus vidas, desde su universo de significados. Esta decisión metodológica entiende que los significados que las nuevas y viejas tecnologías tienen para los jóvenes están ligados a sus contextos sociales, a las prácticas en su cotidianidad, a los imaginarios sobre su condición de jóvenes y sobre el futuro.

La técnica utilizada ha sido la entrevista cualitativa, semiestructurada y con impronta etnográfica, es decir, una entrevista en la que si bien se sigue una pauta previamente elaborada, se adopta el tipo de escucha y de apertura propias de la entrevista en profundidad (GUBER, 1991, 2001). Además de las entrevistas se utilizó la observación en distintas instancias de la vida de la institución educativa y las rutinas de estos jóvenes en ella.

La pauta de entrevista abordó tres dimensiones de exploración:

1. Dimensiones espaciales y temporales de la cotidianidad: se exploran aquí las representaciones y prácticas acerca de las TIC en relación a las rutinas de trabajo y/o estudio, ocio y tiempo libre.

2. Dimensión de la sociabilidad: usos individuales y compartidos; relaciones sociales y vínculos de los jóvenes con la familia, amigos, compañeros de estudio.

3. Representaciones e imaginarios sobre las TIC y su potencial como instrumento de mejora de las condiciones de vida de los jóvenes a corto, mediano y largo plazo.

Estas dimensiones se separaron operativamente para la ejecución de la investigación, pero ellas están profundamente interconectadas y así se presentarán en la exposición de los resultados.

\section{Discusión de algunos resultados}

\section{Claves interpretativas del significado de los usos y las prácticas}

\footnotetext{
${ }^{8}$ Sus estudiantes provienen de zonas rurales de Lavalleja, Canelones, Florida y Rocha. Se entrevistó a la totalidad de estudiantes que estaban cursando el bachillerato en la Escuela en ese momento. Los jóvenes entrevistados asisten en régimen de internado de lunes a viernes y regresan a sus casas en el fin de semana. En cuanto a la dedicación laboral y colaboración con las tareas del predio familiar de los diez jóvenes entrevistados, existe una marcada diferencia en torno al tiempo que cada uno le dedica a dichas actividades: seis colaboran activamente en las actividades agropecuarias que constituyen el sustento de sus familias. Dos trabajan los fines de semana como empleados de ferias vecinales en atención al público. Dos no colaboran con las actividades agropecuarias de sus predios familiares y finalmente, una de las jóvenes no trabaja ni colabora en ninguna de las tareas anteriormente mencionadas.
} 
Los significados de las nuevas tecnologías de la comunicación, los usos y las prácticas en los jóvenes rurales entrevistados están ligados a una situación vital muy diferente a las de los jóvenes que viven en Montevideo, la capital del país, y a las expectativas y representaciones que estos jóvenes tienen sobre el futuro.

Como afirman VÁSQUEZ WIEDEMAN y VALLEJOS QUILODRÁN (2014) "las expectativas de integración globalizante se constituyen en imperativos y marcan la pauta para un desarraigo sostenido en la conectividad permanente y la hibridación" (p.93). Entonces, el hecho de mudarse a la ciudad no es considerado un fin en sí mismo sino un medio para la consecución de objetivos certeramente trazados. Los usos y significados que las TIC adquieren para este grupo de entrevistados es fundamentalmente instrumental: son un modo de mantener el contacto con el lugar de origen y de mantener los vínculos afectivos ligados al hogar y a los amigos, al mismo tiempo que nutre la nostalgia de un entorno natural del que se está alejado. Las tecnologías de la comunicación funcionan así como extensiones del hogar, conectan la interioridad y exterioridad, una relación instalada en la cultura de la vida cotidiana que corresponde a lo que SILVERSTONE define como fenómenos transicionales (SILVERSTONE 1996). Para estos jóvenes el celular adquiere el mismo significado que advertía WINOCUR (2008) en su investigación sobre esa tecnología:

(...) es clave para mantener la cohesión imaginaria de estos espacios seguros donde habitan nuestras certezas, porque nos permite exorcizar a los fantasmas de la otredad, cuando nos cubre bajo el manto protector de estar siempre comunicados con los nuestros (p.177). sostienen que:

No obstante, VÁSQUEZ WIEDEMAN y VALLEJOS QUILODRÁN (2014)

(...) se puede advertir una modificación en las dinámicas relacionales que caracterizaban el campo simbólico de la ruralidad, que ha definido, en términos de Bourdieu, la conformación de un nuevo habitus que, en este caso, se expresa en la tendencia al desarraigo y al extrañamiento respecto a sus rasgos originarios, todo esto entendido como propiedad simbólica del campo p.94).

Se comienza entonces a entretejer un panorama que desencadena cierta tensión reflejada en la necesidad de alejarse de la zona de confort, del terruño, de la morada, para comenzar a materializar las posibilidades de un verdadero desarrollo personal. El desprenderse de los afectos de toda la vida, de lo cotidiano, propiciaría la oportunidad de enfrentarse a desafíos que solo los territorios desconocidos pueden ofrecer. El medio rural es visualizado por estos jóvenes como la tierra firme ya conquistada, la ciudad, en cambio, como el territorio a conquistar. Es este último el escenario ideal para forjar su independencia y retornar "al pago", al lugar de origen, con los conocimientos adquiridos para mejorar su realidad y la de los suyos. Se manifiestan de este modo, sentimientos de ambigüedad (en torno a un inminente desarraigo motivado por la proximidad de la emigración a la ciudad), y por otro lado, la expectativa de mejora de vida que conlleva ese cambio temporal.

"Me gustaría trabajar mismo donde tanto trabajo pasaron mis abuelos para tener esas hectáreas y hacerlas producir, tener animalitos, como ellos siempre tenían, tienen, viste. Tal vez que pueda crecer pero mantener eso, más o menos como siempre, eso me gustaría mucho." (Mónica, 18 años, Canelones). 
El carácter transitorio de la migración a la ciudad en estos jóvenes, se enmarca en el concepto de "migración de retorno" (VÁSQUEZ WIEDEMAN y VALLEJOS QUILODRÁN 2014; OIT 2012, p.96). En este tipo de migración, la acción migratoria prevé un posterior retorno al lugar de origen. Se diferencia así de la "migración circular", que se define por un constante ir y venir de las personas desde sus lugares de origen a sus lugares de estudio y trabajo (p.96).

La idea de cambiar de entorno de vida, de mudarse a la ciudad, se presenta a través de las narrativas de los jóvenes cargada de ambigüedad. Manifiestan tanto esperanza como preocupación asociada a la dificultad que representa adaptarse a los cambios. De algún modo, se maneja una concepción idílica del campo, vinculada a la tranquilidad, a la libertad, opuesta a lo caótico de la realidad urbana. Se plantean ideas arquetípicas de ambos contextos, dicotomías que retroalimentan distintos tipos de sentimientos que se extienden a sus proyecciones de vida y que se ensamblan con los usos que les atribuyen a las TIC:

"Yo pienso usar las redes sociales, usar el celular, llamarlos. A mi madre le gusta que la llame todos los días porque se preocupa por nosotros, obvio, y voy a estar más conectado a las redes sociales, capaz, a lo que voy a estar lejos de ellos. A mí me cuesta mucho pensar que voy a estar lejos del campo, es mi espacio personal donde uno puede estar tranquilo, pensar lo que quieras, tenés libertad, silencio, no escuchás nada, ni un ruido de motor, a veces, no es como en la ciudad que siempre estás escuchando ruidos, es una de las partes difíciles que veo, pero creo me puedo adaptar" (Armando , 17 años, Florida).

"Los primeros meses creo que el teléfono va a ser fundamental para mí porque no sé ubicarme en Montevideo, soy de lo peor, entonces dije "lo primero que voy a hacer es recargarme $\$ 1000$ al “coso" y andar con el gps pero sacándo eso, creo que va a ser lo mismo o voy a tener hasta menos tiempo para mirar televisión, por ejemplo. (Mariela, 18 años Canelones)"

En casos como el de Antonio (15 años, Canelones), quien no cuenta con conectividad en su predio, se visualiza la idea de un mayor acceso futuro a Internet como beneficioso. La desigualdad en términos de acceso a comunicación y información es percibida por parte del joven como una desventaja. En la comparación con sus pares, la brecha digital, le provoca cierta incomodidad. Para él, existe una clara relación entre un mayor acceso a internet y un panorama más alentador en cuanto a oportunidades.

El contacto con el mundo urbano se percibe como camino para el ascenso social en tanto permite la formación universitaria. El uso de las pantallas está ligado así al aprendizaje, al ámbito educativo y a la posibilidad de mantenerse en continuidad con el hogar. Sin embargo, en el discurso de las entrevistas, esta valoración positiva coexiste con una visión más crítica cuando se considera la relación con la comunidad y la familia.

Los conceptos de hogar y comunidad se entrelazan nuevamente con las ideas del retorno, la nostalgia por el pago y el temor al desarraigo por parte de algunos de los jóvenes entrevistados. En este sentido, SILVERSTONE (2004) elabora un concepto de comunidad que permite comprender estos sentimientos y relaciones conflictivas:

En un momento una observación benévola y neutral sobre la vida aldeana (... ) un instante después, el núcleo de un lamento por la pérdida de todo 
lo que se percibe como bueno y verdadero. Soñamos con la comunidad. Con los elementos comunes y las realidades compartidas que la apuntalan (p.157).

En sintonía con esta relación con la comunidad se encuentra la idea del hogar como ámbito que trasciende el espacio físico, dotado de una carga psíquica y emocional: "Hogar como un lugar de refugio. Un lugar tan facilitador como opresivo. Un lugar con límites que hay que definir y defender. Un lugar de regreso. Un lugar desde el cual contemplar el mundo. Privado. Personal. Interior. Conocido. Mío" (SILVERSTONE, 2004, pp.144-145).

Pero el hogar es también fruto de la dialéctica entre el adentro y el afuera de lo público y lo privado, de lo consciente y lo inconsciente y los medios son agentes centralmente involucrados en esta dinámica (SILVERSTONE 2004).

Los medios y artefactos digitales posibilitan entonces el llevar parte de la esfera más íntima hacia donde nos encontremos, independientemente de las razones que hayan motivado el alejamiento de nuestras moradas originales. SILVERSTONE (2004) se pregunta al respecto:

¿Y cuándo no podemos volver a casa? Y cuándo estamos en movimiento, desplazados por las guerras, la política o el deseo de una vida mejor? Con nuestros medios podemos llevar con nosotros algo del hogar (...) Las historias del hogar corren como venas a lo largo del cuerpo social y esas historias ya no están libres de los medios" (p.149-150).

Y de modo más contundente afirma: "Todavía necesitamos los mitos del eterno retorno; y los medios son una de sus fuentes decisivas (p.155).

\section{Tecnologías de la comunicación, familia y comunidad.}

En lo que refiere a aspectos de interacción y cohesión social, para nuestros entrevistados la tecnología no se concibe necesariamente como una ventaja. La auténtica comunicación se favorece a través del diálogo directo y presencial con el vecino. Esto se evidencia en expresiones de melancolía en relación a la forma idiosincrática de relacionarse en el medio rural, cuando la comunicación no se encontraba originalmente mediada por las TIC. Es en este sentido que las tecnologías de la información y la comunicación se ven asociadas a una sensación de eventual amenaza que pone en peligro a la estabilidad de las relaciones de cordialidad y cercanía, percibidas como propias de la vinculación entre la gente de campo. La democratización del acceso a las tecnologías se proyecta, en la percepción de estos jóvenes, como un panorama de despersonalización que atenta contra los usos y costumbres de sus comunidades, a la vez que resulta perturbadora por su influencia negativa en el ámbito familiar:

"Y, con la tecnología, yo veo que se pierde un poco las costumbres del campo y los gurises con la tecnología buscan otras cosas, capaz que los incentivan a otras cosas, no sé, eso va en uno también" (Armando, 17 años, Canelones).

"La tecnología cada vez va a estar más presente, no va a haber una persona que no tenga una computadora en la casa con internet. Cada vez va a ser peor porque va a haber menos comunicación en la casa, en vez de preguntarse cómo les fue en el día, van a estar todo el día con el celular”. (Deborah, 18 años, Canelones).

"Era muy común de ir a pedirle algo al vecino y pasar dos o tres horas hablando con él, y hasta más, a veces, y capaz que perdés el día de trabajo, pero pasas el día con los vecinos, que allá en el Gardel, somos casi todos familia. 
Entonces, ta, y últimamente se está perdiendo esa costumbre y es todo por whatsapp. Hay que no perder eso, eso depende de las personas pero yo creo que de aquí a dos años, la comunicación se va a perder" (Mariela, 17 años Canelones).

"Le está haciendo mal (a su hermano menor) porque en el liceo le está yendo horrible porque no deja el celular. Llega y lo primero que agarra es el celular y no ayuda en casa, no hace nada, está solo con el celular, entonces están todo el día diciéndole "Juan, tenés que dejar eso" para que ayude un poco. (...) Mi padrastro también está con el celular. Y mi mamá le dice” dejá eso que eso es para los más jóvenes" y están ahí discutiendo" (Juana, 17 años, Canelones).

En este sentido, Winocur y Sánchez Vilela (2016) remiten a la noción de la violencia simbólica que ejercen los artefactos digitales sobre el habitus doméstico. Aunque las autoras se expresan en relación a la computadora, es posible establecer una analogía con el empleo del celular en tanto que éste también :

(...) constituye el símbolo emblemático de una nueva forma de vida necesaria para el futuro y el ascenso social, al mismo tiempo representa la pérdida de cierto "modo" de hacer las cosas (...) cuando comienza a mediar en las relaciones familiares, a cambiar las prácticas sociales y perturbar el orden establecido" (p.136).

Así, las rutinas familiares mediadas por las TIC, el empleo del celular específicamente, aparece como una actividad dotada de sentimientos contradictorios. Se presenta como un dispositivo que conflictúa con determinados ritmos o rutinas familiares que serían más deseables $\mathrm{y}$, al mismo tiempo se revela útil, con un uso lúdico intergeneracional, como un instrumento de proximidad afectiva, como lo manifiestan los testimonios siguientes:

"Me aleja mucho del mundo. Porque si yo no estuviera con el celular capaz que podría estar compartiendo momentos con mis primos o algo, pero como el día perfecto para ellos también es con el celular, preferimos estar con el celular antes de tomar unos mates o algo, son peor que yo con el celular." (Daniela 18 años).

“¿Sabés a qué jugamos mucho? El "Preguntados”, ese. A mí me gusta y a ella [su abuela]... vos sabés que se lo mostré un día y le quedó gustando. Y a veces, competimos una contra la otra, se sienta una de cada lado, ella en su teléfono y yo en el mío. A veces decimos "Che abuela, ¿vamos a jugar al Preguntados?" "Bueno, dale, me dice, y jugamos hasta que una de las dos gane" (Mónica, 18 años, Canelones).

\section{La relación con las rutinas de trabajo, estudio y tiempo libre.}

La naturaleza del contexto rural es percibida como el espacio deseado y opuesto al espacio online. Ello se hace explícito en las representaciones del ocio y del tiempo libre, en las que las nuevas tecnologías, en particular el celular, se perciben como distorsivas y se contraponen a la comunicación directa. Así, en las descripciones de un día de ocio o un día divertido, "estar desconectado" es central. Las TIC son reconocidas nuevamente con un rol solo instrumental, ya sea para concertar un encuentro o identificar una ubicación: 
"Para mí, los mejores días libres serían tipo un cumpleaños de la familia que compartimos lindos momentos entre todos. Cuando nos juntamos así la familia, no prendemos la tele, cuando chica, a veces iba y papá me decía: “no, hay visitas y no podés prender la tele" como eso que te inculcan los padres, a veces, y que hasta el día de hoy agradezco, que me hayan enseñado eso" (Mariela, 18 años, Canelones).

"Para mí, un día libre libre perfecto sería, ir a alguna criolla, pasar bien con amigos, haciendo lo que a mí me gusta, alguna prueba de rienda, participar en juegos de campo con caballos. (Antonio 15 años, Canelones).

"Levantarme en casa, salir con mis padres a pasear, estar con mis amigas, tomar mate, visitar a la abuela. En algún momento que estamos conversando, todos juntos no uso el celular, pero ponele que dejemos de conversar un rato, me pongo a contestar los whatsapp o entro a las redes, Facebook o Instagram. Pero a veces, me quedo sin internet y no pasa nada". (Verónica 17 años, Florida)

En la cultura audiovisual de los jóvenes rurales, la radio y la televisión lineal ocupan un lugar central. La primera acompaña las tareas rurales, es el medio por el que circulan las noticias de interés local, las que refieren a eventos y fiestas tradicionales. En las prácticas de estos jóvenes, la radio se inserta como un telón de fondo de las rutinas de trabajo de sus familias y aparece exclusivamente en esos momentos. La escucha radial es una práctica heredada como tradición familiar y se percibe como práctica compartida:

"Cuando vamos a trabajar en el invernáculo, la radio es algo común, prenderla para hacer bochinche, porque es como que nos sentimos solitarios ahí y prendemos la radio del Tala para hacer bochinche. (...) La gente del programa como que te cambia el humor. Ah! y fundamental para papá, a las seis de la mañana escuchar el programa de campo. Siempre hablan sobre los productores chicos, de las fomentos y siempre llevan a alguien para que hablen sobre algún tema y papá siempre me explica alguna cosa que no entiendo. A mí lo que más me gusta es un programa a las 11 que hay en radio Tala, que es más de bobadas, y a papá también le gusta y ahí comentamos de lo que hablan y eso, como que no es solo escuchar la radio, también charlamos de lo que dicen, ¿no? (Mariela, 18 años, Canelones).

"Escuchamos. En la mañana. La Radio Continental, que la escucha mi padre, y nosotros la escuchamos también y por la tarde está la 1600 AM. Y son programas que pasan música tipo folklore y hablan de las fechas de las criollas y te informan también de alguna noticia" (Antonio, 15 años, Florida)

En cuanto al uso de la televisión, la mitad de los jóvenes entrevistados cuenta con acceso a TV cable en sus hogares. Durante la semana que se encuentran en la Escuela Agraria, a través de la computadora miran capítulos de telenovelas en internet. Sin embargo, ver televisión sigue siendo para estos jóvenes una actividad fundamentalmente social y familiar. En el siguiente testimonio se manifiesta la ritualidad compartida en torno a ver un episodio de Game of Thrones:

"Se podría decir que el primer episodio lleva toda una preparación, como un ritual (...) y nos sentamos todos y prendemos la tele unos minutos antes para no perderse nada. El ritual, que le decimos con mi padre, es que si empieza a la diez, prácticamente a las nueve se paraliza el mundo. Se prepara la mesa para comer algo, una pizza, cerrar todo para que no entre ni un rayo de luz, que se pueda ver la tele perfecta, pedir una pizza que obviamente la pizza siempre llega tarde, que es mágico. Empieza a las diez, la pizza llega diez y cuarto, es mágico. Y el ritual sería 
ese, tomar una cerveza con mi padre y estar al pendiente, que no pase nada. (Federico, 17 años, Canelones)

El carácter casi litúrgico que el joven le otorga al momento de compartir su serie favorita, da cuenta de lo que para Silverstone(2004) representa la importancia de los medios en el seno familiar. Su relevancia reside entonces en el "poder que supuestamente ejercen sobre nosotros en casa. Pueden tanto quebrantar como resguardar el santuario" (p153). En esa dirección de valoración del ámbito doméstico y del hogar (SÁNCHEZ VILELA, 2016) se encuentran los relatos en los que la instancia de mirar películas son percibidos como una oportunidad de compartir en familia:

"Somos mucho de mirar tele y películas de cowboys y esas cosas. En vacaciones, a eso de las seis de la tarde, por ahí tomamos mate, mamá hace algo, un picadillo o algo, nos sentamos en el comedor y nos ponemos a mirar una película de cowboys o una graciosa que hubiera en la tele" (Deborah, 18 años, Florida).

"Y mi madre alguna novela y a veces nos colgamos, los dos. No tenemos los mismos gustos pero miramos. Ahora está mirando Kosem, la Sultana o algo de eso y después el programa uruguayo En foco también nos interesa a mí y a mi padre, te explica mucho, aprendés, ves nuevos lugares que no conoces, conoces historias también. $Y$ después alguna película que nos guste a los dos, tratamos de conseguir alguna, tenemos gustos parecidos, casi siempre de acción, o alguna de antes, Cantinflas le gustaba y eso, pero no se ve mucho ahora las películas de esas" (Alex, 17 años. Zona rural de Florida).

Al analizar el proceso acceso-uso-apropiación de las TIC autores como Camacho (2001) se alejan de un planteo lineal y proponen considerarlo como un círculo virtuoso en el cual el usuario se apropia socialmente de Internet, al incorporar en su quehacer diario el uso de la red y ser capaz de distinguir cuando las herramientas tecnológicas, combinadas con otros instrumentos, se tornan relevantes para la resolución de problemas cotidianos y cuando no. Se habla entonces, de un proceso no automatizado sino estratégico, consciente y hasta cierto punto, planificado. Este tipo de uso al que refiere el autor, es también apreciable en las narrativas de nuestros entrevistados, en el sentido de adecuación a la circunstancia:

"Cuando estoy trabajando no soy de usar el celular pero si tengo que comunicarme con mi padre, que es mi mano de apoyo, si tengo algún problema en el campo, lo utilizo, si. Y para sacar fotos que queda grabado el contraste de un animal muerto, sacás una foto, identificás bien el animal para que quede la fecha y hora y después la paso por la red de whatsapp al dueño del establecimiento, y se le pone el número de la caravana y eso. O hay gente que a veces te corta los alambrados para llevarte ganado y tenés que sacarle fotos, identificar para que vean que lo encontraste cortado, es la prueba del crimen, queda todo grabado" (Armando, 17 años, Florida).

"El celular lo usamos a veces para comunicarnos porque si tenemos que salir al monte, porque mi padre es peón rural, para comunicarnos, a ver si estamos bien, porque son 2000 hectáreas donde estamos, divididas en muchos campos, pero días de viento, a mi padre, no le gusta el viento, entonces me pregunta si estoy bien, o si encontramos un bicho muerto o abichado, nos pasamos una foto, o nos avisamos, o 
pasamos el número de caravana para ver qué bicho fue el que se murió". (Tamara ,16 años, Florida).

"Los fines de semana, nos repartimos las tareas, si uno tiene que salir a recorrer las ovejas en época de parición, uno hace eso, el otro le da de comer a los bichos, nos repartimos las tareas, es más fácil. El celular lo llevo por las dudas por si hay alguna complicación me comunico con mi padre. (Deborah, 18 años, Florida).

Así, el celular se inserta en la dinámica de la vida diaria para determinadas acciones prácticas y utilitarias, pero también como herramienta que ameniza las labores del campo:

"Llego los viernes y me cambio y salgo a (... ) recorrer animales, los campos, alambrar si hay que alambrar (...) Y con el celular pongo la música, unos buenos folclores, como que me inspira más a hacer las cosas, o cuando salgo a andar a caballo a recorrer los campos algún folclorito, alguna musiquita jaja. (Mónica, 18 años, Canelones).

En cuanto a las rutinas de estudio, los jóvenes, reconocen la ventaja que otorgan las TIC, específicamente la computadora o celular con acceso a internet, en cuanto a la rápida obtención de la información. No obstante, las reflexiones que se desprenden en sus relatos, dejan entrever una relación de ambigüedad y tensión entre estas tecnologías y lo que ellos consideran la adquisición de aprendizajes significativos. Mayoritariamente, la computadora es de cierto modo menospreciada, al compararla con los libros, estableciéndose una relación binaria casi de oposición más que de complementariedad. A diferencia de su contraparte tecnológico, el libro es concebido como un objeto cuyo abordaje y dominio implican esfuerzo intelectual, concentración y dedicación, procesos que para estos estudiantes se asocian a la idea de retención de saberes y la confiabilidad.

Una aseveración constante en los relatos de los jóvenes es que la facilidad que proporciona la computadora con acceso a internet, no necesariamente favorece el aprendizaje profundo. A nivel general, se evidencia la preferencia de metodologías tradicionales de enseñanza asociadas al esfuerzo y la constancia por parte de los estudiantes, ambos valores, tradicional e históricamente asociados a la etapa previa a la aparición de la computadora. En contrapartida, emerge la noción del libro como el objeto por excelencia, de mayor confiabilidad y que propicia la focalización en la tarea, a diferencia de la navegación en la computadora, que incita a la dispersión.

Se aprecia también, cierta cuota de nostalgia relacionada a la forma de aprender de la niñez, donde surge, por ejemplo, la técnica del dictado del maestro, quien se presenta como guía y mediador irremplazable en los procesos de enseñanza y aprendizaje:

"En la escuela, la maestra me traía los libros, me hacía leer y estudiar y hacer resúmenes, pero, yo que sé, estaba más bueno porque uno ha perdido mucho con el tema de la computadora, que se acostumbra mucho a lo más fácil y ha perdido mucho eso de los libros, como que las bibliotecas están más paradas, ha cambiado mucho." (Mónica, 18 años, Canelones).

"...Yo aprendí mucho más yo con el estilo del maestro Ramón que mi hermana estudiando con la computadora, con las tablets porque yo cuando estaba en cuarto me dictabas algo y te lo escribía y a mi hermana le cuesta escribir" (Mariela, 18 años Canelones). 
“...Y ahí ya se usaban más los libros y se razonaba más porque cuando buscabas algo en un libro tenés que razonarlo para entenderlo y después hacerlo. Para mi es mejor el libro, porque te ayuda a razonar para entender uno mismo porque la computadora por más que te ayude, en algunos casos te perjudica también porque no terminas entendiendo, lo único que hacés es copiar" (Tamara, 16 años, Florida).

El acceso a las TIC no se traduce directamente en la concepción de aprendizajes duraderos, sino que para que esto suceda, se hace imprescindible la presencia mediadora del docente.

\section{Conclusiones}

Las significaciones de las tecnologías de la comunicación para este grupo de jóvenes rurales están ligadas a las expectativas y representaciones que tienen sobre el futuro. Como hemos señalado, al indagar en esa dimensión, se identifican dos campos de sentido: por un lado, el inminente traslado a la ciudad, como lugar de tránsito donde obtener formación y experiencia y por otro, en una proyección temporal más amplia, el retorno a lugar de origen, cargados de ese conocimiento.

A través de los distintos relatos recabados, la idea del bienestar que otorga lo conocido, la sensación de la protección que brinda el hogar, se contrapone a cierto sentimiento de aprensión asociado al futuro. Se constata la tendencia de estos jóvenes rurales a ceñirse a sus entornos originales lo que les permite anclar parte de su emotividad a un ambiente resguardado y seguro, a un refugio. El amor por la "querencia" se manifiesta como la irrefrenable tendencia de volver, una vez completados sus estudios, para reencontrar la seguridad de lo que les es propio y resignificar sus espacios.

La noción del regreso al pago surge recurrentemente en varios de los testimonios de los jóvenes, quienes conciben su acercamiento a la ciudad como un mero proceso transitorio e instrumental, necesario para obtener una titulación que les permita optimizar la calidad de vida en sus lugares de origen.

Como afirman Vásquez Wiedeman y Vallejos Quilodrán (2014) en su estudio sobre las expectativas de futuro de los jóvenes rurales chilenos:

(...) existen fuerzas centrífugas originadas en el seno mismo de la cultura de estos agroterritorios, que expulsan a los jóvenes fuera de sus localidades de origen, pero que, sin embargo, fuerzas centrípetas tales como la familia, el apego a la cultura, las expectativas de desarrollo profesional, etcétera, atraen a los individuos de vuelta hacia su origen (pp. 97-98).

En este plano, marcado por la ambivalencia de emociones, la conexión con el núcleo familiar cobra vital importancia y ese es el principal sentido de uso de las TIC. Se trata de familias que, independientemente de su composición, están muy presentes en varios aspectos de la vida de estos jóvenes, fundamentalmente en el acompañamiento a las trayectorias educativas. Esto se constató en las distintas etapas de observación en la institución, donde fue posible confirmar la presencia de padres y familiares en diversas instancias convocadas por la Escuela Agraria. Se trata de padres, abuelos y tíos que en varios casos, han tenido que realizar grandes 
sacrificios para sustentarse. Es pertinente resaltar, que los jóvenes protagonistas de este estudio, constituyen, en la inmensa mayoría de los casos, la primera generación en culminar estudios secundarios y continuar con su formación terciaria. De cierta manera, son destinatarios de los sueños y esperanzas que se forjan a partir de la ilusión de sus familias.

Las relaciones que se establecen en torno a las TIC, se presentan así, de un modo variable y de forma ambigua. Por un lado, el alejarse de estas tecnologías permite a los jóvenes una reconexión con el entorno y consigo mismos. Por el otro, posibilitan el retomar contacto con seres queridos y difuminar de cierta manera las distancias físicas. La ambigüedad latente en estas situaciones trae aparejada un halo de conflicto. Se trata de un sentimiento de incomodidad recurrente en los usuarios actuales de las TIC. Conservar y proteger la comunión con el entorno natural, y permanece en el mundo on line de forma equilibrada se presentan como escenarios difíciles de reconciliar. ¿Cómo es posible para estos jóvenes establecer real contacto con su contexto original natural "descontaminado" si la adopción de las TIC significa ser absorbido o por lo menos aproximado a lo contrario? Por otro lado, ¿cómo se concibe un despegue real de los límites trazados por sus entornos originales sin el empleo de las TIC y las oportunidades que brindan? Las respuestas no se encuentran en un plano superficial de análisis ya que subyacen, en parte, en las dos caras de la moneda que se observa en sus discursos.

Paradójicamente, a través de la percepción de los propios jóvenes se replica la idea de que es desde la ciudad de donde provienen las oportunidades de progreso. Parece ser necesario acudir a lo urbano como forma de optimizar y validar lo rural, por momentos concebido "(... ) como una instancia arcaica a ser superada a través del desarrollo modernizante" (GONZÁLEZ CANGAS, 2003, p.5).

Nos encontramos ante ideas arquetípicas de ambos contextos en estos jóvenes, dicotomías que retroalimentan distintos tipos de sentimientos que se extienden a sus proyecciones de vida y que se ensamblan con los usos y sentidos que les atribuyen a las TIC.

La idea de tradición es un fuerte componente constituyente del sentido de la identidad de estos jóvenes y se extiende a su proyección de vida futura. Se destaca notoriamente la forma en que los jóvenes enmarcados en este estudio, manifiestan un gran compromiso por mantener vivas las costumbres del campo. Esto se refleja en su activa participación en festividades criollas y sociedades nativistas.

Estos jóvenes dan lugar a una reinterpretación de la tradición mediada por las tecnologías que se materializa también en sus proyecciones de futuro. La fotografía del "gaucho a caballo con celular" se reitera en el paisaje campestre, escenario que ellos también componen. Los jóvenes rurales ven en las tecnologías de la comunicación un instrumento que favorece la comunicación interpersonal para extender invitaciones a eventos gauchescos o para colaborar en las tareas del campo y consolidar la construcción de su autopercepción. Las TIC tienen lugar en su presente y también en su visión de futuro. Sin embargo, el carácter instrumental es el predominante y son utilizadas en el entendido de que colaboran en la consecución de determinados objetivos y en tanto no obstaculicen ámbitos de la vida personal, que ellos consideran esenciales e irremplazables. 


\section{REFERENCIAS}

CALVO, J. J. (coord.). Atlas sociodemográfico y de la desigualdad en Uruguay. Jóvenes, demografía, educación, mercado laboral, y emancipación. Montevideo, Trilce, 2014. Disponible en

<http://www.ine.gub.uy/documents/10181/34017/Atlas fasciculo 4 jovenes.pdf /c19f7b00-c004-4e35-ace5-8d38ded573dd > Consultado 26 febrero 2018.

CAMACHO, K. ¿Internet: Una herramienta para el cambio social? México, FLACSO, 2001.Disponible en< https://www.sulabatsu.com/wp-content/uploads/2010internet herramienta cambio social.pdf>_Consultado 8 marzo 2016.

CASAMAYOU, A. Apuntes para una agenda lo rural. uy.En: Anuario Flor de Ceibo. Informe 2012. Comisión Sectorial de Enseñanza. Comisión Sectorial de Extensión y Actividades en el Medio Comisión Sectorial de Investigación Científica. Universidad de la Republica, Junio 2013 p. 267-276. Disponible en $<$ http://www.flordeceibo.edu.uy/sites/default/files/AnuarioFC 2012 completo .pdf> Consultado 8 marzo 2016.

CASAMAYOU, A. Apropiación(es): aportes desde la sistematización y la teoría. En: RIVOIR, A. (coord.). Tecnologías digitales en sociedad: análisis empíricos y reflexiones teóricas. Montevideo. Universidad de la República. Facultad de Ciencias Sociales, p.15-21, 2016. Disponible en: <http://observatic.edu.uy/wpcontent/uploads/2019/08/tecnologias-digitales-en-sociedad-observatic.pdf Consultado 20 setiembre 2019>

DODEL, M.; AGUIRRE, F. Principales resultados de la encuesta WIP+Disto Uy 2017. Montevideo: Universidad Católica del Uruguay, 2018.

DURSTON, J. La situación de la juventud rural en América Latina - Invisibilidad y estereotipos. División de Desarrollo Social

CEPAL., 1996. Disponible en < http://www.fao.org/3/x5633s/x5633s01.htm > Consultado 10 febrero 2018.

DURSTON, J. Juventud rural en Brasil y México. Reduciendo la invisibilidad. Ponencia presentada en XX Congreso de la Asociación Latinoamericana de Sociología. Agosto-setiembre, 1997.

DURSTON, J. Juventud rural en Brasil y México. Reduciendo la invisibilidad. CEPAL, 1998. Disponible en

< https://repositorio.cepal.org/bitstream/handle/11362/19783/S9850408 es.pd f?sequence=1\&isAllowed $=\mathrm{y}>$ Consultado 30 marzo 2018 .

ESCOSTEGUY, A. C. (coord.). As tecnologías de comunicaçâo no cotidiano de familias rurais, Santa Cruz do Sul, EDUNISC, 2019. 
EUTIC (2019). Disponible en<https://www.gub.uy/agencia-gobierno-electronicosociedad-informacion-conocimiento/comunicacion/noticias/resultadospreliminares-eutic-2019> Consultado en 14 de febrero 2020.

FEIXA, C.; OLIART, P. (coords.). Juvenopedia. Mapeo de la Juventudes Iberoamericanas. Barcelona. Ned Publicaciones, 2016.

GARCÍA CANCLINI, N.; URTEAGA, M.; CRUCES, F. (Coords.) Jóvenes, Culturas Urbanas y Redes Digitales, (p.25-42). Barcelona: Fundación Telefónica y Ariel.

GONZÁLEZ CANGAS, Y. Juventud rural: trayectorias teóricas y dilemas identitarios. Nueva Antropología, 19(63), 153-175, 2003.

GUBER, R. El salvaje metropolitano. Reconstrucción del conocimiento social en el trabajo de campo. Buenos Aires, Argentina: Editorial Paidós, 1991.

GUBER, R. La entrevista etnográfica o el arte de la "no directividad", La etnografía. Método, campo y reflexividad. Bogotá, Colombia: Grupo Editorial Norma, p. 30-40, 2001.

GRUPO RADAR (2017), El Perfil del Internauta Uruguayo, 2017.

GRUPO RADAR (2018), El Perfil del Internauta Uruguayo 2018.

HALL, S.; JEFFERSON, T. Rituales de resistencia. Subculturas juveniles en la Gran Bretaña de posguerra. La Plata: Ediciones de Universidad de La Plata, 2011. ITU (2017). Measuring the Information Society Report 2017.v. 1. Disponible en $<$ https://www.itu.int/en/ITU

D/Statistics/Documents/publications/misr2017/MISR2017_Volume1.pdf >Consultado 14 de junio de 2020.

MARCOS ARÉVALO, J. La tradición, el patrimonio y la identidad. Revista de estudios extremeños. p. 925-956, 2004.

MORDUCHOWICZ, R. La generación multimedia. Significados, consumos y prácticas culturales de los jóvenes. Buenos Aires, Paidós, 2008.

MORDUCHOWICZ, R. Los adolescentes y las redes sociales.išpi La construcción de la identidad juvenil en Internet. Buenos Aires, FCE, 2012.

REGUILLO, R. Emergencias culturales juveniles. Estrategia del desencanto. Bogotá, Colombia, Editorial Norma, 2000.

REGUILLO, R. (coord). Los jóvenes en México. México FCE/CONACULTA, 2010.

RÜDIGER, E. Fotografia e identidade territorial a partir dos jovens no vale do Rio Pardo-RS. Dissertação (Programa de Pós-Graduação em Desenvolvimento Regional 
- Mestrado e Doutorado, Área de Concentração em Desenvolvimento Regional, Linha de Pesquisa em Território, Planejamento e Sustentabilidade)- Universidade de Santa Cruz do Sul, UNISC, 2016.

SÁNCHEZ VILELA, R. Jóvenes y cultura audiovisual: los nuevos modos de ver televisión, Porto Alegre, Revista Famecos, 2016.

SERRANO, Y. Múltiplos olhares, múltiplas mediações: As representações sociais da ruralidade entre os jovens rurais da microrregião de Santa Cruz do Sul. Tese (Programa de Pós-Graduação em Desenvolvimento Regional (Doutorado), área de concentração em Desenvolvimento Regional, dentro da linha de pesquisa Território, Planejamento e Sustentabilidade- Universidade de Santa Cruz do Sul, UNISC, 2017.

SILVERSTONE, R. ¿Por qué estudiar los medios?. Buenos Aires, Amorrortu. 2004. SUNKEL, G. El acceso de los jóvenes a la cultura en la era digital en América Latina. En Trucco, D; Ullmann, $\mathrm{H}$ (eds.). Juventud: realidades y retos para un desarrollo con igualdad. Santiago, CEPAL, 2015.

VÁSQUEZ WIEDEMAN, C.; Vallejos Quilodrán, D. Migración juvenil rural en la región del Maule, Chile. Expectativas de futuro de la nueva generación. Revista de Ciencias Sociales. v.27 n.35. pág. 91-108. Montevideo, dic. 2014.

WINOCUR, R. El móvil, artefacto ritual para exorcizar la otredad. FLACSO. En Albornoz, M. B.; Cerbino, M. (compiladores) Comunicación, cultura y política Quito, Ecuador, $1^{\mathrm{a}}$. edición:. p.173-189, septiembre, 2008.

WINOCUR, R.; SÁNCHEZ, R. Familias pobres y computadoras. Claroscuros de la apropiación digital. Montevideo. Editorial Planeta, 2016.

Rosario Sánchez Vilela. Doctora en Ciencia Política, Master en Comunicación social. Universidad Católica del Uruguay (UCU). Profesora Titular. Directora del Departamento de Comunicación y de la Maestría en Comunicación. Recepción y Cultura. Investigadora del Sistema Nacional de InvestigadoresANII, Nivel II. Charrúa 2092, Montevideo, Uruguay.rsanchez@ucu.edu.uy; rsanchezvilela@gmail.com

Celeste Borjas. Profesora de Educación Media. Mención inglés. Universidad Católica del Uruguay. Estudiante de la Maestría en Comunicación. Recepción y Cultura de UCU. 


\section{CONTRIBUCIÓN DE CADA AUTOR}

Este artículo forma parte de la investigación de la tesis de Maestría en Comunicación (UCU) de Celeste Borjas, bajo la orientación de Rosario Sánchez Vilela. Por otra parte, integra el proyecto de investigación Jóvenes y cultura audiovisual del que esta última es responsable científica.

Conceituação (Conceptualization) Celeste Borjas-Rosario Sánchez Vilela Curadoria de Dados (Data curation) Celeste Borjas Análise Formal (Formal analysis) Celeste Borjas, Rosario Sánchez Vilela Obtenção de Financiamento (Funding acquisition) No aplica Investigação/Pesquisa (Investigation) Celeste Borjas Metodologia (Methodology) Rosario Sánchez Vilela- Celeste Borjas Administração do Projeto (Project administration) Rosario Sánchez Vilela Recursos (Resources) No aplica Software No aplica Supervisão/orientação (Supervision) Rosario Sánchez Vilela Validação (Validation) No aplica Visualização (Visualization) No aplica Escrita - Primeira Redação (Writing - original draft) Celeste Borjas Escrita - Revisão e Edição (Writing - review \& editing). Rosario Sánchez Vilela 